A Sadi

Nagoya Math. J.

Vol. 126 (1992), 103-124

\title{
SOME TYPES OF REGULARITY FOR THE DIRICHLET PROBLEM
}

\author{
AMAR SADI
}

The question of whether the existence of a harmonic majorant in a relative neighbourhood of each point of a boundary of a domain $D$ implies the existence of a harmonic majorant in the whole of $D$ has received great attention in recent years and has been dealt with by several authors in different settings. The most general results to date have been achieved in [10] with the Martin boundary. In [9], the author arrives, by independent means, at the conclusions of [10] in the particular case where $D$ is a Lipschitz domain.

In this paper, we answer the question in domains with suitably regular topological frontiers. Our methods rely heavily on the possibility of obtaining an extented-representation for nonnegative superharmonic functions defined near a frontier point. This naturally led to the introduction and the study of new types of regularity for the generalised Dirichlet problem. As well as their suitability in dealing with the question of harmonic majorisation, they present an intrinsic importance as natural extensions of the (classical) regularity. For simplicity reasons, we will treat the finite boundary points and the point at infinity separately.

We start with a type of regularity which, although introduced in a new way, will later be seen to be equivalent to Armitage's strong regularity given in [2].

We first give some conventions concerning the notations.

Unless we specify otherwise, all the sets considered are subsets of $N$-dimensional Euclidean space $R^{N}$ with $N \geq 2$.

Points of $R^{N}$ as well as singletons (i.e. sets consisting of one point) are denoted by a single letter. However, points are, when necessary, expressed in terms of their coordinates. The norm $|\cdot|$ is the Euclidean norm.

For a point $y$ of $R^{N}$ and a positive reel number $r$, the open ball $B(y, r)$ is the set

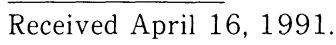




$$
\left\{x \in R^{N}:|x-y|<r\right\}
$$

and the sphere $S(y, r)$ is the set

$$
\left\{x \in R^{N}:|x-y|=r\right\} \text {. }
$$

If, in addition, $R>r$ then the open annulus $A(y, r, R)$ is the set

$$
\left\{x \in R^{N}: r<|x-y|<R\right\} \text {. }
$$

For a subset $E$ of $R^{N}$ is the finite topological boundary of $E$. The frontier Fr $E$ of $E$ is $\partial E$ of $E$ is bounded and $\partial E \cup\{A\}$ if $E$ is unbounded, where $A$ is the point at infinity (i.e. the Alexandroff point). Note therefore that $\operatorname{Fr} E$ is considered as a subset of the compactified Euclidean space $\overline{R^{N}}$.

By a domain we always mean a non-empty connected open subset of $R^{N}$.

The notations $H_{f}, \bar{H}_{f}, \underline{H}_{f}, U_{f}, \ldots$, are standard.

Their exact definition as well as a detailed study of the generalised Dirichlet problem can be found in [8].

Finally, Property (g) refers to the property (g) given in ([8], 1, VIII. 6).

\section{L-regularity}

Let $\Omega$ be a Green open set in $R^{N}, f$ a function on $\operatorname{Fr} \Omega$ resolutive for the Dirichlet problem and $H_{f, \Omega}$ the Dirichlet solution for $f$ in $\Omega$. When there is no risk of confusion, we may write $H_{f}$ instead of $H_{f, \Omega}$.

We recall that a point $y_{0}$ on $\operatorname{Fr} \Omega$ is regular (for $\Omega$ ) if and only if for each real-continuous function $f$ on $\operatorname{Fr} \Omega$,

$$
\lim _{x-y_{0}} H_{f, \Omega}(x)=f\left(y_{0}\right) \quad(x \text { in } \Omega) .
$$

The following theorem is known, at least implicitly.

Theorem A. A point $y_{0}$ on $\partial \Omega$ is regular if and only if (1.1) holds for each nonnegative real continuous function $f$ on $\operatorname{Fr} \Omega$ such that

$$
f \equiv 0 \text { on } B\left(y_{0}, R\right) \cap \operatorname{Fr} \boldsymbol{\Omega}
$$

for some positive real number $R$.

This theorem inspired the following

Definition 1.3. A point $y_{0}$ on $\partial \Omega$ is said to be $L$-regular ( $L$-for 1. s.c.) if (1.1) holds for each nonnegative, extended-real-valued, lower-semi-continuous and resolutive function $f$ satisfying (1.2). 
In the sequel, all functions are supposed to be extended-real-valued unless we specify otherwise. Our first result is a criterion for L-regularity.

THEOREM 1.4. Let $\Omega$ be a Green open set and $y_{0}$ a point on $\partial \Omega$. Then $y_{0}$ is $L$ regular if for each function $f$ on $\operatorname{Fr} \Omega$ such that $\bar{H}_{f}$ is harmonic

$$
\limsup _{x \rightarrow y_{0}} \bar{H}_{f}(x) \leq \limsup _{x \rightarrow y_{0}} f(y) \quad(x \text { in } \Omega, y \text { on } \operatorname{Fr} \Omega) \text {. }
$$

Proof. Suppose that (1.5) holds and let $f$ be a nonnegative, l.s.c., and resolutive function on $\operatorname{Fr} \Omega$ satisfying (1.2). Then

$$
\underset{x \rightarrow y_{0}}{\limsup } \bar{H}_{f}(x) \leq 0 \quad(x \text { in } \Omega) .
$$

Thus (1.1) holds for $f$. Hence $y_{0}$ is $L$-regular.

Now assume that $y_{0}$ is $L$-regular and let $f$ be a function on $\operatorname{Fr} \Omega$ with $\bar{H}_{f}$ harmonic in $\Omega$, and let $\lambda$ be the value of the right hand side of (1.5). If $\lambda=+\infty$, there is nothing to prove. So assume that $\lambda<+\infty$. First, we suppose that $\lambda$ is finite and let $g=f-\lambda$. Then

$$
\limsup _{y \rightarrow y_{0}} g(Y)=\limsup _{y \rightarrow y_{0}} f(\bar{Y})-\lambda=0
$$

and

$$
\underset{x \rightarrow y_{0}}{\limsup } \bar{H}_{g}(X)=\limsup _{x \rightarrow y_{0}} H_{f}(X)-\lambda \text {. }
$$

By (1.6), for each $\varepsilon>0$ there exists $R>0$ such that

$$
g \leq \varepsilon \text { in } B\left(y_{0}, R\right) \cap \operatorname{Fr} \Omega .
$$

Now since $f$ is upper resolutive, so is $g$. Thus there exists a function $u>0$ in the upper family $U_{g}$. Let

$$
F(y)= \begin{cases}0 & \text { for } y \text { in } \overline{B\left(y_{0}, R / 2\right)} \cap \operatorname{Fr} \Omega . \\ \liminf _{x \rightarrow y} u(x) \text { for } y \text { in } \operatorname{Fr} \Omega \backslash \overline{B\left(y_{0}, R / 2\right)} .\end{cases}
$$

Then $F$ is resolutive since $F$ is 1 .s.c., bounded below and $u$ is in $U_{F}$. Since $y_{0}$ is $L$-regular it follows that

$$
\lim _{x-y} H_{F}(x)=0 .
$$

On the other hand, $g \leq \varepsilon+F$ on $\operatorname{Fr} \Omega$ whence

$$
\bar{H}_{g} \leq \varepsilon+\bar{H}_{F}=\varepsilon+H_{F} \text { in } \Omega .
$$

Thus 


$$
\limsup _{x \rightarrow y_{0}} \bar{H}_{g}(x) \leq+\varepsilon+\lim _{x-y_{0}} H_{F}(Y)=\varepsilon .
$$

Using (1.7) and bearing in mind that $\varepsilon$ is arbitrary we get

$$
\limsup _{x \rightarrow y_{0}} \bar{H}_{f}(x) \leq \lambda \text {. }
$$

Now, if $\lambda=-\infty$ then $f$ is continuous at $y_{0}$. For each positive integer $n$, let $f_{n}=$ sup $(f,-n)$. Since $f \leq f_{n}$ for each $n$, and using the preceding argument for $f_{n}$, we have

$$
\limsup _{x \rightarrow y_{0}} \bar{H}_{f}(x) \leq \limsup _{x \rightarrow y_{0}} \bar{H}_{f n}(x) \leq \limsup _{x \rightarrow y_{0}} f_{n}(y)=-n .
$$

Letting $n$ tend to infinity, we get

$$
\limsup _{x \rightarrow y_{0}} \bar{H}_{f}(x) \leq-\infty=\lambda \text {. }
$$

COROLlary 1.8. A point $y_{0}$ on $\partial \Omega$ is L-regular if and only if (1.1) holds for all resolutive functions on $\operatorname{Fr} \Omega$ which are continuous (in the extended sense) at the point $y_{0}$.

Proof. The "if" part is clear, using Definition 1.3. To prove the converse, let $f$ be a resolutive function on $\operatorname{Fr} \Omega, f$ continuous at $y_{0}$, and assume that $y_{0}$ is $L$-regular. By Theorem 1.4.

$$
\liminf _{y \rightarrow y_{0}} f(y)=f\left(y_{0}\right) \leq \liminf _{x \rightarrow y_{0}} H_{f}(x) \leq \limsup _{x \rightarrow y_{0}} H_{f}(x) \leq \limsup _{y \rightarrow y_{0}} f(y)=f\left(y_{0}\right) .
$$

Hence

$$
\lim _{x \rightarrow y_{0}} H_{f}(x)=f\left(y_{0}\right)
$$

Corollary 1.8 and Theorem 1.4 show that the L-regularity, Armitage's strong regularity in [2] and Naim's complete regularity in [13] are all equivalent notions.

Using the last corollary, we give an example of an open set $\Omega$ with point on $\partial \Omega$ which are regular but not $L$-regular. This example was first used in [4] to show that a resolutive function $f$ on $\operatorname{Fr} \Omega$ may be bounded in a relative neighbourhood of a boundary point without the same holding for the function $H_{f, \Omega}$.

EXAMPLE 1.8. For each positive integer $n$, let

$\Omega_{n}=(0,1) \times(1 /(n+1), 1 / n)$

$\alpha_{n}=$ closed segment $\{(x, y): x=0$ and $1 /(n+1) \leq y \leq(1 / n+1 /(n+1)) / 2\}$

and

Let

$$
\Omega=\bigcup_{n=1}^{\infty} \Omega_{n}
$$




$$
f=\sum_{n=1}^{\infty} k_{n} \chi_{n}
$$

where, for each $n, \chi_{n}$ is the characteristic function of $\alpha_{n}$. Since $\alpha_{n}$ is of positive harmonic measure for $\Omega_{n}$, hence for $\Omega$, the constant $k_{n}$ can be chosen so that the Dirichlet solution $H_{f}$ of $f$ in $\Omega$ is equal to $n$ at the centre $P_{n}$ of the rectangle $\Omega_{n}$. Then $H_{f}\left(P_{n}\right)$ tends to infinity as $\left(P_{n}\right)$ tends to the point $P=(1 / 2,0)$ even though $f$ is identically equal to 0 in a neighbourhood of this point. Thus the point $P$ is not $L$-regular for $\Omega$. However the point $P$ is regular for $\Omega$ since $R^{2} \backslash \Omega$ is not thin at $P$.

The preceding argument shows in fact that all the points of the segment $\{(x, y): 0<x<1$ and $y=0\}$ are regular but not $L$-regular for $\Omega$.

\section{2. $B$-regularity}

Definition 2.1. Let $\Omega$ be a Green open set and $y$ a point on $\partial \Omega$. Then $y$ is said to be $B$-regular for $\Omega$ ( $B$ - for bounded) if for each resolutive function $f$ bounded in $B(y, R) \cap \operatorname{Fr} \Omega$ for some $R>0$ the function $H_{f, \Omega}$ is bounded in $B(y, \rho) \cap \Omega$ for some $\rho>0$.

The property that $H_{f, \Omega}$ is bounded near $y$ whenever $f$ is bounded near $y$ will for brevity reasons be denoted by $[\mathrm{PB}]$.

Using Property (g) ([8], 1. VIII, 6) we see that if there exists a neighbourhood $\omega$ of $y$ such that $y$ is $B$-regular for $\omega \cap \Omega$ then $y$ is $B$-regular for $\Omega$.

Note that Example 1.8 provides an example of a boundary point which is regular but not $B$-regular. With few modification (see for instance [13], Section 46) the example also shows that, unlike the set of irregular points, the set of boundary points which are not $B$-regular is neither always polar nor of zero harmonic measure.

Here we prove a simple result that shows that there are points which are $B$-regular but not regular.

Proposition 2.2. Let $D$ be a Green domain and $y$ a point on $\partial D$. Suppose there exists $\rho>0$ such that $B(y, \rho) \cap \partial D$ is polar. Then $y$ is $B$-regular for $D$ (but not regular). In particular any isolated point of $\partial D$ is $B$-regular.

Proof. Let $E$ be set $B(y, \rho) \cap \partial D$. Then $E$ is polar and closed in $B(y, \rho)$ so that $B(y, \rho) \backslash E$ is connected. As $B(y, \rho) \cap D$ is nonempty, it follows that

$$
B(y, \rho) \backslash E \subset D .
$$

Thus 


$$
\operatorname{Fr}(D \cup B(y, \rho)) \cup E=\operatorname{Fr} D .
$$

Now let $f$ be a resolutive function on $\operatorname{Fr} D$ such that $f$ is bounded in a neighbourhood of $y$. Since any lower bounded (resp. upper bounded) superharmonic (resp. subharmonic) function in $D$ has a superharmonic (resp. subharmonic) extension to $D \cup E(=D \cup B)$. It is easy to deduce from the definition of $H_{f, D}$ that $f$ is resolutive for $D \cup B$ and

$$
H_{f, D} \equiv H_{f, D \cup B}
$$

in $D$. Thus, if

$$
F= \begin{cases}f & \text { on } \operatorname{Fr} D \backslash E \\ H_{f}, D \cup_{B} & \text { on } E \text { with } B=B(y, \rho),\end{cases}
$$

then $F$ and $f$ differ only on a polar hence negligeable subset of $\operatorname{Fr} D$. Hence for any $x$ in $D$.

$$
H_{f, D \cup B}(x)=H_{F, D}(x)=H_{f, D}(x) .
$$

As $y$ is in $D \cup B$ then $H_{f}, D \cup B$ is bounded in a neighbourhood $V$ of $y$ and therefore by (2.3) we get $H_{f, D}$ is bounded in $V \cap D$. Thus $Y$ is $B$-regular for $D$. However (2.3) also shows that $H_{f}, D$ does not depend on the value of $f$ at the point $y$ whence $y$ is not regular.

We now give some useful criteria $B$-regularity.

Proposition 2.4. Let $\Omega$ be a Green open set and $y$ a point on $\partial \Omega$. The following are equivalent

(i) $y$ is B-regular.

(ii) $[P B]$ holds for each resolutive function $f$, finite and continuous at $y$.

(iii) $[P B]$ holds for each nonegative, resolutive function $f$ that vanishes in a neighbourhood of $y$.

(iv) $[P B]$ holds for each nonnegative, resolutive and l.s.c. function $f$ that vanishes in a neighbourhood of $y$.

Proof. It is clear that (i) $\rightarrow$ (ii) $\rightarrow$ (iii) $\rightarrow$ (iv) $(\rightarrow$ for implies). We only need proving that (iv) implies (i).

Let $f$ be a nonnegative resolutive function on $\operatorname{Fr} \Omega$ such that $f$ is bounded in $B(y, R) \cap \partial \Omega$ by a constant $M$. Since $f$ is resolutive, there exists a superharmonic function $v$ in the upper family $U_{f}$. By adding a suitable positive constant to $v$ we get a nonnegative function $u$ in $U_{f}$.

Let $F$ be the function 


$$
F(Z)= \begin{cases}0 & \text { for } Z \text { on } \overline{B(y, R / 2)} \cap \partial \Omega \\ \liminf _{x \rightarrow z} u(x) \text { for } Z \text { on } \operatorname{Fr} \Omega \backslash \overline{B(y, R / 2)} .\end{cases}
$$

Then $F$ is nonnegative, 1.s.c. and resolutive since $\bar{H}_{F}=H_{F}$ and $u$ is in $U_{F}$. Thus by (iv), there exists $\rho>0$ and $k>0$ such that

$$
H_{F, \Omega} \leq k \text { in } B(y, \rho) \cap \Omega \text {. }
$$

On the other hand,

$$
f \leq M+F \text { on } \operatorname{Fr} \Omega \text {. }
$$

Hence

$$
\begin{aligned}
H_{f, \Omega} & \leq M+H_{F, \Omega} \text { in } \Omega . \\
& \leq M+k \quad \text { in } B(y, \rho) \cap \Omega .
\end{aligned}
$$

THEoREM 2.5. Let $D$ be a Creen domain and $y$ a point on $\partial D$. Then $y$ is $B$-regular for $D$ if and only if for each neighbourhood $V$ of $y$, there exists a neighbour. hood $V_{0}$ of $y$ such that for any resolutive function $f$ on $\operatorname{Fr} D$ bounded in $V \cap \operatorname{Fr} D$. $H_{f, D}$ is bounded in $V_{0} \cap D$.

Proof. The "if" part of the theorem is clear. We now prove the "only if" part. Let $f$ be a resolutive function, bounded in $V \cap \operatorname{Fr} D$. It is enough to prove the result when $f$ is nonnegative. Then, as seen in the proof of Proposition 2.4, if $W$ is a neighbourhood of $y$ with closure in $V$, there exist a constant $M \geq 0$ and a re. solutive function $F$ on $\operatorname{Fr} D$ such that $F$ vanishes in $W \cap \operatorname{Fr} D$ and $f \leq M+F$ on $\operatorname{Fr} D$. Therefore it is enough to prove the result for any nonnegative and resolutive function that vanishes in a fixed neighbourhood $V$ of $y$. We will do this by contra. diction.

Let $\left(V_{n}\right)$ be a sequence of open neighbourhoods of $y$ such that $\cap V_{n}=\{y\}$. Suppose that for each $V_{n}$, there exist a nonnegative resolutive function $f_{n}$ and a point $y_{n}$ in $V_{n} \cap \partial D$ such that $f_{n} \equiv 0$ in $V \cap \operatorname{Fr} D$ and

$$
\limsup _{x \rightarrow y_{n}} H_{f_{n, l}}(x)=+\infty \text {. }
$$

We normalise the sequence $f_{n}$ by taking

$$
H_{f_{n, \nu}}(x)=1 / n^{2}
$$

where $x_{0}$ is a fixed point in $D$. Let

$$
g_{m}=\sum_{n=1}^{m} f_{n}
$$

and $g=\lim _{m \rightarrow \infty} g_{m}$. 
Since $\left(g_{m}\right)$ is an increasing sequence of resolutive functions, we have

Thus

$$
\bar{H}_{g, D}=\lim _{m-\infty} \bar{H}_{g_{m, D}}=\lim _{m-\infty}\left(\sum_{n=1}^{\mathrm{m}} \bar{H}_{f_{n}}\right) \text {. }
$$

$$
\bar{H}_{g, D}\left(x_{0}\right)=\sum_{n=1}^{\infty} 1 / n^{2}<\infty .
$$

Moreover, using ([8], Theorem 1, VIII, 6, page 110) we have

$$
\bar{H}_{g, D}=\underline{H}_{g, D} \text { in } D
$$

Hence $g$ is nonnegative, resolutive for $D$ and vanishes in $V \cap \operatorname{Fr} D$. On the other hand, since $g \geq f_{n}$ for each $n$ and $\left(y_{n}\right)$ tends to $y$ as $n$ tends to $\infty$ it follows that

$$
\limsup _{x \rightarrow y} H_{g, D}(x) \geq \lim _{n-\infty}\left(\limsup _{x \rightarrow y_{n}} H_{f_{n, D}}(x)=\infty .\right.
$$

This is now impossible since $y$ is $B$-regular and the contradiction establishes Theorem 2.5 .

Note that in the preceding proof the function $g$ may not be bounded on $\operatorname{Fr} D$ even if each function $f_{n}$ is bounded. So (2.6) does not constitute a contradiction to the regularity of $y$ but to its $B$-regularity. Thus the proof does not give the boundedness of $H_{f, D}$ in a fixed neighbourhood of $y$ in $D$ when the $B$-regularity hypothesis is replaced by a regularity one.

\section{A boundary Harnack principle}

Next we use Theorem 2.5 and an argument due to Armitage ([2]) to prove that the notions of $B$-regularity and $L$-regularity are equivalent to boundary Harnack principle.

THEOREM 3.1. Let $D$ be a Green domain, $y$ a point on $\partial D$ and $x_{0}$ a fixed point of $D$. Then $y$ is $B$-regular if and only if for each neighbourhood $V$ of $y$, there exist a positive constant $k$ and a neighbourhood $V_{0}$ of $y$ such

$$
H_{f, D(x)} \leq k H_{f, D}\left(x_{0}\right)
$$

for all $x$ in $V_{0} \cap D$, and every nonnegative, resolutive function $f$ that vanishes on $V \cap \operatorname{Fr} D$.

Proof. The "if" part follow from Proposition 2.4, (iv). To prove the "only if" part, let $L$ be the vector space consisting of all resolutive functions that vanish on $V \cap$ Fr $D$. By Theorem 2.5, there exists a neighbourhood $V_{0}$ of $y$ such that for any $f$ in $L, H_{f, D}$ is bounded in $V_{0} \cap D$. For each $x$ in $V_{0} \cap D$, let 


$$
T_{x}: \mathrm{f} \longmapsto \int_{\mathrm{Fr} D} f(\mathrm{z}) d \mu_{x(z)}=\mathrm{H}_{f, D}(x) .
$$

Then $T_{x}$ is a linear mapping of $L$ into the real line $R$. Moreover, if we define for each function $f$ in $L$.

$$
\|f\|=H_{|f|, D}\left(x_{0}\right)
$$

then. $\|\cdot\|$ is a norm and $L$, provided with this norm, is the vector space $L^{1}(y)$ where $\gamma$ is the restriction of the harmonic measure $\mu_{x_{0}}$ to $\operatorname{Fr} D$. Hence $\operatorname{Fr} D \backslash V$ is a Banach space. Now, for each $f$ in $L$, let

$$
E_{f}=\left\{T_{x}(f): x \text { in } V_{0} \cap D\right\} .
$$

By Theorem 2.5, $E_{f}$ is a bounded subset of $R$. Hence $T_{x}$ is pointwise (or weakly) bounded. On the other hand, using the classical Harnack inequalities, there exists $\lambda>0$ (depending on $x$ but not on $f$ ), such that

$$
\left|H_{f, D}(x)\right| \leq \lambda H_{|f|, D}\left(x_{0}\right)
$$

for all $f$ in $L$. Thus $T_{x}$ is continuous. By the Banach-Steinhauss Theorem, the set $\left(T_{x}: x\right.$ in $\left.V_{0} \cap D\right)$ is therefore equicontinuous so that there exists $k>0$ (independent of $x$ and $f$ ) such that

$$
\left|T_{x}(f)\right| \leq k\|f\|
$$

for all $x$ in $V_{0} \cap D$ and all $f$ in $L$. We now get (3.2) by taking $f$ nonnegative in the last inequality.

Corollary 3.3. Let $D$ be a Green domain, $y$ a point on $\partial D$ and $x_{0}$ a point in $D$. Then $y$ is B-regular if and only if given a neighbourhood $V$ of $y$ there exist a constant $k \geq 0$ and a neighbourhood $V_{0}$ of $y$ such that

$$
\mu_{x(E)} \leq k \mu_{x_{0}}(E)
$$

for all $y$ in $V_{0} \cap D$ and any $\mu_{x_{0}}$-measurable subset $E$ of $\operatorname{Fr} D \backslash V$.

Proof. Suppose that $y$ is $B$-regular let $E$ be a $\mu_{x_{0}}$-measurable subset of $\operatorname{Fr} D \backslash V$. Then $E$ is $\mu_{x}$-measurable for all $x$ in $D$ and (3.4) follows from (3.2) by taking $f=\chi_{E}$.

Now assume that (3.4) holds and let $f$ be a nonnegative resolutive function vanishing in $V \cap \operatorname{Fr} D$. Then for all $x$ in $V_{0} \cap D$, we have

$$
H_{f, D}(x)=\int_{\mathrm{Fr} D \backslash V} f(z) d \mu_{x}(x) \leq \int_{\mathrm{Fr} D \backslash V} f(z) k d^{\mu} x_{0}(z)=k H_{f, D}\left(x_{0}\right) .
$$


Thus $y$ is $B$-regular by Theorem 3.1.

We now deal with the connection between $B$ - and $L$-regularity. As seen in Section 2, the two notions are distinct. However using Theorem 3.1 we are able to show that the set of $B$-regular points and the set of $L$-regular points differ only by a set of irregular points.

Theorem 3.5. Let $D$ be a Green domain, $y$ a point on $\partial D, x_{0}$ a point in $D$ and $V$ a neighbourhood of $y$. The following are equivalent

(i) $y$ is L-regular

(ii) $y$ is regular and B-regular

(iii) For each $\varepsilon>0$ there exists a neighbourhood $V_{0}$ of $y$ such that

$$
H_{f, D}(x) \leq \varepsilon H_{f, D}\left(x_{0}\right)
$$

for all $x$ in $V_{0} \cap D$ and all $f \geq 0$, resolutive with $f \equiv 0$ in $V \cap \operatorname{Fr} D$.

Proof. It is clear that (i) implies (ii). It is easy to prove that (iii) implies (i) as follows. If (3.6) holds, then as $\varepsilon$ is arbitrary, it follows that $H_{f, D}(x)$ tends to 0 as $x$ tends to $y$. Thus $y$ is $L$-regular by definition.

We now prove that (ii) implies (iii). Suppose that $y$ is $B$-regular. By Theorem 3.1 there exist $\kappa>0$ and a bounded neighbourhood $V_{1}$ of $y$ such that $\bar{V}_{1} \subset V$ and (3.6) holds with $\kappa$ instead of $\varepsilon$ and $V_{1}$ instead of $V_{0}$. Let $V_{z}$ be a neighbourhood of $y$ such that $\bar{V}_{2} \subset V_{1}, g$ be the function equal to $\kappa$ on $\partial V_{z} \cap D$ and vanishing everywhere else and finally let $F$ be the function equal to $H_{f, D}$ on $\partial V_{2} \cap D$ and to zero everywhere else. Thus, using

$$
\partial\left(V_{2} \cap D\right) \subset\left(\bar{V}_{2} \cap \partial D\right) \cup\left(\partial V_{2} \cap D\right)
$$

it comes that

$$
F \leq g H_{f, D}\left(x_{0}\right) \text { on } \partial\left(V_{2} \cap D\right) .
$$

Thus, for $x$ in $V_{2} \cap D$

$$
H_{f, D(x)}=H_{F, V_{2} \cap D}(x) \leq H_{g, V_{2} \cap D}(x) . H_{f, D}\left(x_{0}\right) .
$$

Now $g$ is bounded, resolutive for $V_{2} \cap D$ and $g$ is continuous and vanishes at $y$. As $y$ is regular for $D$ it is also regular for $V_{2} \cap D$ whence

$$
\lim _{x-y} H_{g, V_{2} \cap D}(x)=0 .
$$

Thus for any $\varepsilon>0$ there exists a neighbourhood $V_{3}$ of $y$ such that

$$
H_{g, V_{2} \cap D} \leq \varepsilon \text { in } V_{3} \cap D .
$$


Hence, if $V_{0}=V_{2} \cap V_{3}$ then for all $x$ in $V_{0} \cap D$, we have

$$
H_{f, D}(x) \leq \varepsilon H_{f, D}\left(x_{0}\right) .
$$

Note that using Theorem 3.5 we get a criterion for $L$-regularity in terms of harmonic measures similar to the one given in Corollary 3.3 for the $B$. regularity.

\section{Examples of $L$ - and $B$-regular domains}

\section{Potentials and $B$-regularity.}

THEOREM B. Let $D$ be a Green domain any $y$ a point on $\partial D$. Suppose that for each neighbourhood $V$ of $y$ there exists a neighbourhood $V_{0}$ of $y$ with the property that for any Radon measure $\mu>0$ on $D$ concentrated on $D \backslash V$ (i.e. $\mu(V)=0$ ), its Green potential is either identically equal to infinity in $D$ or bounded in $V_{0} \cap D$. Then $y$ is B.regular.

This theorem is easily deduced from results in [6].

\section{Geometrical conditions.}

The next result shows that if $\partial D$ is "nice" near $y$, then $y$ is $B$-regular.

Definition 4.1. Let $D$ be a domain and $y$ a point on $\partial D$. Then $D$ belongs to the class $N(y)$ if there exists an arbitrary small neighbourhood $W$ of $y$ such that

(i) $W \cap D$ is a union of a finite number of domains $D_{i}$.

(ii) For each domain $D_{i}$ there exists a ball $B_{i}$ containing $y$ such that $B_{i} \cap$ $\partial D \subset W$ and the inverse of $B_{i} \cap \bar{D}_{i}$ with respect to $\partial B_{1}$ is in $D_{i}$.

This is a slight generalisation of a notion that was first introduced by Brelot in [5]. The expression " $W$ arbitrary small" is taken in the sense that for any $\varepsilon>0$, there exists a neighbourhood $W$ of $y$ such that $W \subset B(y, \varepsilon)$.

One proves, along the same lines as Brelot, that such domains satisfy a Harnack Principle in a neighbourhood of the point $y$ and therefore, in particular, $y$ is $B$-regular. More precisely we have the following

Theorem C. Let $D$ be a Green domain, $y$ a point on $\partial D$ such that $D$ is in $N(y)$, and $A$ a fixed point in $D$. Then, there exist $k>0$ and an open ball $B(y, R)$ such that

$$
H_{f, D} \leq k H_{f, D}(A) \text { in } B(y, R) \cap D,
$$

for each nonnegative, resolutive function $f$ with $f \equiv 0$ on $W \cap \operatorname{Fr} D$. where $W$ is the 
open set given in Definition 4.1. It follows, in particular, that $y$ is B-regular.

We now give a geometrical condition for $L$-regularity. Essentially the same result has been proved in [2].

THEOREM D. Let $\Omega$ be a Green open set and $y$ a point on $\partial \Omega$. Suppose there exists an open neighbourhood $W$ of $y$ such that $W \cap \Omega$ is a union of a finite number of Lipschitz domains. Then $y$ is L-regular.

\section{The Alexandroff point}

We will now define the notions of $B$ - and $B$-regularity of the Alexandroff point $\mathscr{A}$. Both are introduced as extensions of their respective counterpart for the finite boundary points.

Definition 5.1. Let $\Omega$ be an unbounded Green open subset of $R^{N}$. We say that $\mathscr{A}$ is $B$-regular for $\Omega$ if for each resolutive function $f$ that is bounded in $\left\{\overline{R^{N}} \backslash B(0, R)\right\} \cap \operatorname{Fr} \Omega$ for some $R>0$ the function $H_{f, \Omega}$ is bounded in $\left\{R^{N} \backslash B(0, \rho)\right\} \cap \Omega$ for some $\rho>0$.

Similarly, we define the $L$-regularity of $\mathscr{A}$. We say that $\mathscr{A}$ is $L$-regular for $\Omega$ if

$$
\lim _{x \rightarrow \infty} H_{f, \Omega}(x)=0
$$

for all nonnegative, extended-real-valued, lower-semi-continuous and resolutive function $f$ such that $f \equiv 0$ in $\left\{\overline{R^{N}} \backslash B(0, R) \cap \operatorname{Fr} \Omega\right.$ for some $R>0$.

With basically the same proofs, we can check that most results on $B$ - and $L$-regular finite boundary points have analogues when we consider $\mathscr{A}$. Of particular interest are analogues of Theorem 3.1 and Theorem 3.5.

As an example of the type of theorems we get, we give the following analogue of Theorem 3.5.

THEOREM 5.2. Let $D$ be an unbounded Green domain, $x_{0}$ a point in $D$ and $R>0$. The following are equivalent

(i) $\mathscr{A}$ is L-regular.

(ii) $\mathscr{A}$ is $B$-regular and regular.

(iii) For each $\varepsilon>0$, there exists $\rho>0$ such that

$$
\begin{aligned}
& H_{f, D}(x) \leq \varepsilon H_{f, D}\left(x_{0}\right) \\
& \text { for all } x \text { in }\left\{R^{N} \backslash \overline{B(0, \rho)}\right\} \cap D \text { and all } f \geq 0 \text {, resolutive with } f \equiv 0 \text { in } \\
& \left\{\overline{\left.R^{N} \backslash \overline{B(0, \mathrm{R})}\right\}} \cap \operatorname{Fr} D\right. \text {. }
\end{aligned}
$$


Naturally the question arises as to whether the inversion preserves $B$ - or $L$-regularity.

Theorem 5.3. Let $D$ be a Green domain in $R^{N}$ and $y$ a point on $\operatorname{Fr} D$, Let $D^{\prime}$ and $y^{\prime}$ be the image of $D$ and $y$ respectively under an inversion of centre 0 .

Then

(i) $y$ is B-regular for $D$ if and only if $y^{\prime}$ is B-regular for $D^{\prime}$.

(ii) When $N=2$ or $y \neq \mathscr{A}$, then $y$ is L-regular for $D$ if and only if $y^{\prime}$ is L-regular for $y^{\prime}$.

Proof. Suppose that $y^{\prime}$ is $B$-regular for $D^{\prime}$ and let $f \geq 0$ be a resolutive function on $\operatorname{Fr} D$ such that $f \in 0$ in a neighbourhood $V$ of $y, V$ being of the form $\overline{R^{N}} \backslash \overline{B(0, R)}$ if $y=\mathscr{A}$. Then if $f^{\prime}$ is the image of $f$ under the Kelvin transform associated with the inversion and letting $f^{\prime}\left(y^{\prime}\right)=0$, we have

$$
H_{f, D}=\left(H_{f^{\prime}, D^{\prime}}\right)^{\prime} .
$$

On the other hand if $y^{\prime}$ is $B$-regular for $D^{\prime}$ and $x_{0}{ }^{\prime}$ is a point in $D^{\prime}$ then there exists a neighbourhood $V_{1}$ of $y^{\prime}$ and $\lambda>0$ (both independent on $f$ ) such that

$$
H_{f^{\prime}, D^{\prime}} \leq \lambda H_{f^{\prime}, D^{\prime}}\left(x_{0}{ }^{\prime}\right)
$$

in $V_{1} \cap D^{\prime}$. Thus taking the Kelvin transform we get

$$
H_{f, D} \leq\left(\lambda H_{f^{\prime}, D^{\prime}}\left(x_{0}{ }^{\prime}\right)\right)^{\prime}=\lambda H_{f, D}\left(x_{0}\right)
$$

in $\left(V_{1}\right)^{\prime} \cap D$. As $\left(V_{1}\right)^{\prime}$ is a neighbourhood of $y$ it follows that $y$ is $B$-regular for $D$.

Part (ii) follows from (i) and the fact that the inversion preserves regularity when $N=2$ or $y \neq \mathscr{A}$.

When $N>2$ and $y=\mathscr{A}$, then the inverse of $y$ may not be $L$-regular even if $y$ is. For instance, if $D$ is the complement of the closed unit ball then $\mathscr{A}$ is $L$-regular for $D$. However, the image 0 of $\mathscr{A}$ under an inversion of centre 0 is an isolated point of $\operatorname{Fr} D^{\prime}$. Thus 0 is not $L$-regular for $D^{\prime}$.

Remark 5.4. A straightforward use of the definition of $B$-regularity yields the following result.

Let $\Omega$ be a Green open set, $y$ a point on $\partial \Omega$ and $x$ a point distinct from $y$. Let $\Omega^{\prime}$ and $y^{\prime}$ be the image of $\Omega$ and $y$ respectively under an inversion of centre $x$. Then $y$ is $B$-regular for $\Omega$ if and only if $y^{\prime}$ is $B$-regular for $\Omega^{\prime}$. 


\section{Local $B$-regularity}

Definition 6.1. Let $\Omega$ be an open subset of $R^{N}$ and $y$ a point on $\operatorname{Fr} \Omega$. Then, $y$ is said to be locally $B$-regular (lB-regular) for $\Omega$ if there exists a sequence $\left(\rho_{n}\right)$ of positive real numbers converging to 0 such that for all $n, y$ is $B$-regular for $B(y$, $\left.\rho_{n}\right) \cap \Omega$ if $y \in \partial \Omega$ and for $\left\{R^{N \backslash \overline{B\left(0,1 / \rho_{n}\right)}}\right\} \cap \Omega$ if $y=\mathscr{A}$.

We say that $\Omega$ is $l B$-regular if each point $y$ of $\operatorname{Fr} \Omega$ is $l B$-regular. Observe that using Property $(\mathrm{g})$, it is a simple exercise to prove that given a point $Q$ on $\operatorname{Fr} \Omega$ then $\Omega$ is $l B$-regular if and only if each point of $\operatorname{Fr} \Omega$ is $l B$-regular.

Finally, by a neighbourhood of $\mathscr{A}$ we mean a set of the form $\overline{R^{N}} \backslash K$ where $K$ is a compact subset of $R^{N}$.

An immediate example of an $l B$.regular domain is the unit ball $B(0,1)$ or the set $B(0,1) \backslash\{0\}$. In fact, for any $\rho>0$ and any point $y$ on $S(0,1), B(y, \rho) \cap$ $B(0,1)=\omega$, say, is a Lipschitz domain. Hence $y$ is $B$-regular for $\omega$ by Theorem 4. D. Also, it is clear that 0 is $l B$-regular for $B(0,1) \backslash\{0\}$.

Now let $D$ be a Green domain, $f$ a resolutive function on $\operatorname{Fr} D$, and $y$ a point on $\partial D$ such that $f$ is bounded in $\mathrm{B}(y, R) \cap \partial D$ for some $R>0$. For each $\rho$ such $0<\rho<R$, let

$$
\omega=B(y, \rho) \cap D
$$

and $F$ be a function on $\partial w$ equal to $f$ on $\partial \omega \cap \partial D$ and to $\mathrm{H}_{f, D}$ on $\partial \omega \cap D$. Then

$$
H_{F, w}=\mathrm{H}_{f, D} \text { in } \omega .
$$

Suppose that $y$ is $l B$-regular for $D$. Then $H_{F, \omega}$ is bounded near $y$ in $\omega$ for some suitable choice of $\rho$. Hence it follows from (6.2) that $H_{f, D}$ is bounded near $y$ in $D$. Thus $y$ is $B$-regular for $D$. However, we are unable to solve the converse question, i. e. "if a point is $B$-regular, is it $l B$-regular?"

Definition 6.1 also implies that if $V$ is some neighbourhood of $y$, then $y$ is $l B$-regular for $D$ if and only if $y$ is $l B$-regular for $V \cap D$.

\section{Examples of $l B$-regular domains.}

(i) A Lipschitz domain is $l B$-regular.

In fact, if $D$ is a Lipschitz domain and $y$ is a point on $\partial D$, there exists an arbitrary small neighbourhood $U_{n}$ of $y$ such that $U_{n} \cap D$ is a Lipschitz domain (see for example [7], page 281). Thus $y$ is $B$-regular for $U_{n} \cap D$. Hence, using (6.2), $y$ is $B$-regular for $B\left(0, \rho_{n}\right) \cap \mathrm{D}$, where $U_{n} \subset B\left(0, \rho_{n}\right)$. Hence $y$ is $l B$-regular for $D$. 
(ii) If $D$ is a domain and $y$ is a point on $\partial D$ such that $D$ is in $N(y)$, then $y$ is $l B$-regular for $D$ by Theorem $\mathrm{C}$.

(iii) A non-tangentially-accessible domain is $l B$-regular (see [11], Theorems 5.1 and 3.11$)$.

THeorem 6.3. Let $\Omega$ be an $l B$-regular open subset of $R^{N}$ and $y$ a point on $\partial \Omega$. If $0<\rho<R$, and $u$ is a nomnegative superharmonic function in $B(y, R) \cap \Omega$, then there exists a superharmonic function $u^{*}$ in $\Omega$ such that $u^{*} \equiv u$ in $B(y, \rho) \cap \Omega$ and $u^{*}$ is bounded below if $\Omega$ is bounded or $N \geq 3$.

$$
\text { Proof. Let } \begin{aligned}
\omega & =B(y, R) \cap \Omega \\
W & =(B(y, R) \backslash \overline{B(y, \rho)}\} \cap \Omega(=\omega \backslash \overline{B(y, \rho)})
\end{aligned}
$$

and $f$ be the function equal to $u$ on $\partial W \cap \omega$ and to 0 everywhere else. Then $u$ is in the upper family $U_{f, w}$ since $u$ is bounded below and liminf $u \geq f$ on $\partial W$. Moreover $f$ is lower semi-continuous and bounded below on $\partial W$. Thus $f$ is resolutive for $W$ and $u \geq H_{f, w}$ in $W$. On the other hand, simple topological arguments show that

$$
\partial W \cap \omega=\partial B(y, \rho) \cap \omega .
$$

Thus each point $z_{0}$ of $\partial W \cap \omega$ is regular for $W$ and $W$ is not thin at $z_{0}$. Hence

$$
u\left(z_{0}\right)=\liminf _{x \rightarrow z_{0}(x \in w)} u(x) \geq \liminf _{x \rightarrow z_{0}(x \in w)}(x) \geq \liminf _{z \rightarrow z_{0}(z \in \partial w)} f(z)=u\left(z_{0}\right) .
$$

Thus the function $u_{1}$ equal to $u$ in $\omega \backslash W$ and to $H_{f, w}$ in $W$ is superharmonic in $\omega$. Further, since $\Omega$ is $l B$-regular, $u_{1}$ is bounded in $A\left(y, \rho_{1}, \rho_{2}\right) \cap \Omega$ where $\rho<\rho_{1} \rho_{2}$ $<R$. Let $k$ be an upper bound of $u_{1}$ on $S\left(y, \rho_{1}\right) \cap \omega$ and $\sigma$ the fundamental superharmonic function with pole at $y$. If $a$ and $b$ are constants such that

$$
a \sigma+b=k+1 \text { on } S\left(y, \rho_{1}\right) \cap \omega
$$

and

$$
a \sigma+b=-1 \text { on } S\left(y, \rho_{2}\right) \cap \omega,
$$

then the function $a \sigma+b=v$ say, is such that

$$
\begin{aligned}
& v>u_{1} \text { on } S\left(y, \rho_{1}\right) \cap \omega \text { and } \\
& v<u_{1} \text { on } S\left(y, \rho_{2}\right) \cap \omega .
\end{aligned}
$$

As $u_{1}$ and $v$ are both continuous in $W$, it follows that for each point $z$ of $S\left(y, \rho_{1}\right)$ $\cap \omega$ (resp. $\left.S\left(y, \rho_{2}\right) \cap \omega\right)$, there exists a neighbourhood of $z$ where $v>u_{1}$ (resp. $v<u_{1}$ ) holds. 
Thus the function $u^{*}$ which is equal to $u_{1}$ in $\dot{B}\left(y, \rho_{1}\right) \cap \Omega$, to $\min \left(u_{1}, v\right)$ in $\overline{A\left(y, \rho_{1}, \rho_{2}\right)} \cap \Omega$ and to $v$ in $\Omega \backslash \mathrm{B}\left(y, \rho_{2}\right)$ is superharmonic in $\Omega$ and clearly satisfies the required properties.

Remark 6.6. It is important to note that the hypothesis of $l B$-regularity for $\Omega$ in Theorem 6.3 can be considerably weakened. In fact, from the proof we see that it is enough to suppose that for some $\rho_{1}>0$ such that $\rho<\rho_{1}<R$, all points of $S\left(y, \rho_{1}\right) \cap \partial \Omega$ are $l B$-regular for $\Omega$ so that $u_{1}$ be bounded on $S\left(y, \rho_{1}\right) \cap \omega$.

In the sequel any reference to Theorem 6.3 should be taken in this general context.

\section{Positive harmonic majorisation}

For any open set $\Omega$, let $\mathrm{HM}^{+}(\Omega)$ be the set of subharmonic functions $s$ in $\Omega$ such that $s$ has a nonnegative harmonic majorant in $\Omega$. This class of functions was originally for half-spaces by Solomencev in [15]. Different results concerning $\mathrm{HM}^{+}(\Omega)$ have been established since then (see for instance [3] and [14]).

Note that $\mathrm{HM}^{+}(\Omega)$ is also the class of all subharmonic functions $s$ in $\Omega$ such that $s^{+}$has a harmonic majorant in $\Omega$.

Before giving our main theorem of this section, we recall a definition. A family $\left(\Omega_{\lambda}\right)_{\lambda \in \Lambda}$ of subsets of $R^{N}$ is called an open cover of $\operatorname{Fr} \Omega$ if each set $\Omega_{\lambda}$ is open, each point of $\partial \Omega$ is in some $\Omega_{\lambda}$ and when $\Omega$ is unbounded (i.e. $\mathscr{A} \in \operatorname{Fr} \Omega$ ) then at least one set $\Omega_{\lambda}$ is of the form $R^{\mathrm{N}} \backslash K$ where $K$ is a compact subset of $R^{N}$.

THEOREM 7.1. Let $\Omega$ be an lB-regular Green open subset of $R^{\mathrm{N}}$ and $s$ be a subharmonic function in $\Omega$. Suppose there exists an open cover $\left(\Omega_{\lambda}\right)_{\lambda \in \Lambda}$ of $\operatorname{Fr} \Omega$ such that for each $\lambda$, the function $s$ is in $\mathrm{HM}^{+}\left(\Omega_{\lambda} \cap \Omega\right)$. Then $s$ belongs to $\mathrm{HM}^{+}(\Omega)$.

Proof. Suppose first that $\Omega$ is bounded. Since $\operatorname{Fr} \Omega$ is compact in $R^{N}$, it can be covered by a finite number of open balls $\left\{B\left(y_{i}, \rho_{i}\right), i \leq n\right\}$ such that for all $i$, $B\left(y_{i}, 2 \rho_{i}\right)$ is in some $\Omega_{\lambda}$. For each $i$, let $h_{i}$ be a nonnegative harmonic majorant of $s$ in $B\left(y_{1}, 2 \rho_{i}\right) \cap \Omega$. By Theorem 6.3, there exists a superharmonic function $u_{i}$ in $\Omega$ such that $u_{i}$ is bounded below in $\Omega$ by a constant $k_{1} \leq 0$, say, and such that $u_{i}=h_{i}$ in $B\left(y_{i}, \rho_{i}\right) \cap \Omega$. Let

$$
u=\sum_{i=1}^{n}\left(u_{i}-k_{i}\right)
$$

Then $u$ is a nonnegative superharmonic in $\Omega$ and in $B\left(y_{i}, \rho_{i}\right) \cap \Omega$, we have

$$
s \leq h_{i} \leq u_{i}-k_{i} \leq u \text {. }
$$

The Maximum Principle now implies that $s \leq u$ in $\Omega$. But if $\operatorname{Ghm}(u, \Omega)$ is the 
greatest harmonic minorant of $u$ in $\Omega$, then we have

$\operatorname{Ghm}(u, \Omega)=\sup \{g: g$ is subharmonic in $\Omega$ and $g \leq u$ in $\Omega\}$.

Thus, using $s \leq u$ in $\Omega$, we get

$$
s \leq \mathrm{Ghm}(u, \Omega) .
$$

Hence $\operatorname{Ghm}(u, \Omega)$ is a nonnegative harmonic majorant of $s$ in $\Omega$.

Now suppose that $R^{N} \neq \bar{\Omega}$ is nonempty. By Remark 5.4 it follows that if $y \in \partial \Omega$ is $l B$-regular for $\Omega$ then its image $y^{\prime}$ under an inversion of centre 0 and radius $\varepsilon$, where 0 is a point in $R^{N} \backslash \bar{\Omega}$ is $l B$-regular for the inverse $\Omega^{\prime}$ of $\Omega$. Thus all points of $\partial \Omega^{\prime} \leq\{0\}$ are $l B$-regular for $\Omega^{\prime}$. Moreover, for any point $y^{\prime}$ of $\partial \Omega^{\prime}$, including 0 , there exists a neighbourhood $\Omega^{\prime}{ }_{\lambda}$ of $y^{\prime}$ such that $s^{\prime}\left(s^{\prime}\right.$ is the image of $s$ the Kelvin transform corresponding to the above inversion) has a nonnegative harmonic majorant in $\Omega_{\lambda}^{\prime} \cap \Omega$. Further $\Omega^{\prime}$ is bounded. The first part of the proof and Remark 6.6 now show that $s^{\prime}$ has a nonnegative harmonic majorant in $\Omega^{\prime}$. By inversion it follows that $s$ has a nonnegative harmonic majorant in $\Omega$. Now if $\Omega$ is any $l B$-regular Green open set, we let $B(0, R)$ be a ball with closure in $\Omega$ and set $\Omega^{\prime}=\Omega \backslash \overline{B(0, R)}$. Then $\Omega^{\prime}$ is also $l B$-regular and there exists an open cover $\left(w_{\alpha}\right)$ of $\operatorname{Fr} \Omega^{\prime}$ such that $\mathrm{s} \in H M^{+}\left(\omega_{\alpha} \Omega\right)$ so that $\mathrm{s} \in H M^{+}\left(\Omega^{\prime}\right)$ by the second part of this proof. There are now several ways of concluding that $\mathrm{s} \in H M^{+}(\Omega)$. Without recourse to the Riesz representation theorem, we may use

Lemma 7.2. Let $\Omega$ be a Green open set and $B(0, R)$ a ball with closure in $\Omega$. For each function $u$ superharmonic in $\Omega \backslash \overline{B(0, R)}$ and $k>0$, there exist a function $v$ superharmonic in $\Omega$ and $\lambda>0$ such that

$$
u=v-\lambda G(0, .)
$$

in $\Omega \backslash B(0, R+k)$, where $G(0, \cdot)$ is the Green function for $\Omega$ with pole at 0 .

Proof. We may assume that $u$ is real continuous in the closed annulus $A\left(0, R+k^{\prime}, R+k\right)$ with $0<k^{\prime}<k$. Let $\sigma$ be the fundamental superharmonic function with pole at $0 ; k_{1}, k_{2}$ and $\lambda$ be real numbers and define

$$
V^{*}=\left\{\begin{array}{l}
k_{1} \sigma+k_{2} \text { in } B\left(0, R+k^{\prime}\right) \\
\min \left(k_{1} \sigma+k_{2} u+\lambda \sigma\right) \text { in } A\left(0, R+k^{\prime}, R+k\right) \\
u+\lambda \sigma \text { in } \Omega \backslash B(0, R+k) .
\end{array}\right.
$$

Then $V^{*}$ is superharmonic in $\Omega$ provided $k_{1}, k_{2}$ and $\lambda$ satisfy

$$
\begin{gathered}
k_{1} \geqslant 0 \\
k_{1} \sigma(R+k)+k_{2}=\varepsilon+\sup \{u(x)+\lambda \sigma(x): X \in S(0, R+k)\} \\
k_{1} \sigma\left(R+k^{\prime}\right)+k_{2}=-\varepsilon+\inf \left\{u(x)+\lambda \sigma(x): X \in S\left(0, R+k^{\prime}\right)\right\}
\end{gathered}
$$


for some $\varepsilon>0$. From the last two equalities it comes

$$
\begin{aligned}
& k_{1}=\lambda+[2 \varepsilon+(\sup \{u(x): x \in S(0, R+k)\} \\
& \left.-\inf \left\{u(x): x \in S\left(0, R+k^{\prime}\right)\right\}\right]\left[\sigma(R+k)-\sigma\left(R+k^{\prime}\right)\right]^{-1} .
\end{aligned}
$$

Thus with $\lambda$ large enough, we get $k_{1} \geqslant 0$.

Now let $h$ be the greatest harmonic minorant of $\sigma$ in $\Omega$. Then

$$
G(0, \cdot)=\sigma-h
$$

so that $u=v^{*}-\lambda(h+G(0, \cdot))$

$$
=v^{*}-\lambda h+\lambda G(0, \cdot) \text {. }
$$

Thus the required function $v$ is given by $v=v^{*}-\lambda h$.

We now finish the proof of Theorem 7.1 by applying Lemma 7.2 to a positive harmonic majorant $h$ of $s$ in $\Omega$. We get a positive superharmonic function $u$ in $\Omega$ and $\lambda>0$ such that

$$
s \leqslant h=u-\lambda G(0, \cdot) \leqslant u
$$

in $\Omega \backslash B(0, R+k)$ ( $k$ is such that $B(0, R+2 k)$ is in $\Omega$ ). Thus $s$ has a positive superharmonic majorant in $\Omega$ and the result follows.

Theorems of the type 7.1 play an important pole in the study of several problems in the theory of functions. In particular, extensive use of these theorems has been made in investigating the Multiplicative Cousin problem (see for instance [1] and [16]). Later, we will consider an application of Theorem 7.1 to a "new" type of Dirichlet problem.

We now give an example to illustrate Theorem 7.1.

Example 7.3. Let $D$ be the half-space $\left\{(x, y)\right.$ in $\left.R^{2}: y>0\right\}$ and $s(x, y)$ $=x^{+}$. Then $D$ is $l B$-regular and $s$ is a positive subharmonic function in $D$ which is bounded near each point of $\partial D$. However, we will show that $s$ has no harmonic majorant in $D$. From Theorem 7.1 we then deduce that there is no neighbourhood $\Omega_{j}$ of $\{A\}$ such that $s$ has a harmonic majorant in $\Omega_{j} \cap D$. However, if we let

$$
D^{\prime}=D \backslash\{(x, y): x=0 \text { and } y \geq \alpha\}
$$

where $\alpha$ is a constant, then $s$ has a harmonic majorant in $D^{\prime}$.

To prove that $s$ has no harmonic majorant in $D$, we use a criterion due to Kuran ([12]). For $z=(x, y)$ in $D$, let

$$
I(y)=\int_{R} S(z) /\left[x^{2}+(y+1)^{2}\right] d x=\int_{0}^{\infty} x /\left[x^{2}+(y+1)^{2}\right] d x
$$


A simple computation shows that

$$
I(y)=\infty \text { for each } y .
$$

Kuran's criterion now implies the non-existence of a harmonic majorant of $s$ in $D$.

Now for each point $Q$ of $\partial D^{\prime} \backslash\{(0, \alpha)\}$, there exists $R>0$ such that for any $\rho>0$ with $\rho<R$, the set $B(Q, \rho) \cap D^{\prime}$ is either a half-ball or the union of two half-balls. Since a half-ball is Lipschitz it follows that $Q$ is $l B$-regular for $D^{\prime}$. Moreover, for each point $Q$ of $\partial D^{\prime}$ and $R>0, s$ has a harmonic majorant in $B(Q, R) \cap D^{\prime}$ since, in fact, $s$ is bounded there. On the other hand, if $R^{\prime}>\alpha$ and $R^{\prime}>0$, then $s$ has a harmonic majorant in $\left(R^{N} \backslash \overline{B\left(0, R^{\prime}\right)}\right) \cap D^{\prime}$ since in fact $s$ is harmonic there. Thus, by Theorem 7.1, $s$ has a harmonic majorant in $D^{\prime}$.

\section{A Dirichlet problem in $l B$-regular domains}

An extention of the classical Dirichlet problem was studied on the Martin boundary by Gauthier and Goldstein ([10]). Here we redefine it for the topological boundary.

Let $D$ be a Green domain in $R^{N}, N \geq 2$ and $f$ a continuous extended realvalued function on $\operatorname{Fr} D$. A harmonic function $h$ in $D$ is aids to be an inner (Dirichlet) solution for $f$ in $D$ if $h(x)$ has limit $f(y)$ at all points $y$ of $\operatorname{Fr} D$. Any such a function $h$ will be denoted by $I_{f, D}$.

This is a natural extension of the classical Dirichlet problem. Thus the regularity (in the generalised Dirichlet problem (or PWB) sense) of $D$ is necessary for the existence of an inner Dirichlet solution for each continuous function $f$. However, unlike the classical Dirichlet problem, the regularity of $D$ is not sufficient here.

Note also that when an inner Dirichlet solution exists, it is not necessarily unique. For instance, if $D$ is the unit ball $B(0,1), Q$ is a point on $S(0.1), f=\sigma_{Q}$ is the fundamental superharmonic function with pole at $\Omega$, and $K_{Q}$ is the Poisson kernel at $\Omega$, then $\sigma_{Q}+\lambda K_{Q}$ is an inner solution for $f$ in $D$ for all $\lambda \geq 0$.

THeOREM 8.1. Let $D$ be an lB-regular domain such that $R^{N} \backslash \bar{D}$ is not empty and $D$ is regular for the Generalised Dirichlet problem. Let $f$ be a continuous extendedreal-valued function on $\operatorname{Fr} D$. Then an inner Dirichlet solution $I_{f, D}$ exists if and only if the PWB solution $H_{f, D}$ exists.

Proof. Since $D$ is $B$-regular and regular, $D$ is $L$-regular by Theorem. Thus if $H_{f, D}$ exists, then for all $y$ on $\operatorname{Fr} D$

$$
\lim H_{f, D}(x)=f(y)(x \text { in } D)
$$


as $x$ tends to $y$. Hence $H_{f, D}$ is an inner Dirichlet solution for $f$.

Conversely, suppose that an inner Dirichlet solution $I_{f, D}$ exists. If $f \geq 0$, then $I_{f, D}$ is in $U_{f, D}$. Further if $f_{n}=\min (f, n)$, where $n$ is a positive integer, then $f_{n}$ is real-continuous hence resolutive. Thus, using ([8], Theorem 1. VIII, 6, (e)) it comes

$$
0 \leq \bar{H}_{f, D}=\underline{H}_{f, D}<+\infty
$$

Hence the PWB exists.

Suppose now that $f$ is of any sign. Since, $I_{f, D}(x)$ tends to $f(y)$ when $x$ tends to $y$ on $\operatorname{Fr} D$, the function $\left|I_{f, D}\right|$ has a harmonic majorant in some $W \cap D$ where $W$ is a neighbourhood of $y$. For, if $f(y)$ is finite then $\left|I_{f, D}\right|$ is bounded in $W \cap D$ and if $\left|I_{f, D}\right|=+\infty$, then $\left|I_{f, D}\right|= \pm I_{f, D}$ in $W \cap D$. Furthermore, $\left|I_{f, D}\right|$ is subharmonic in $D$. Thus, by Theorem $7.1,\left|I_{f, D}\right|$ has a harmonic majorant $H$ in $D$. Hence, as $x$ tends to a point $y$ on $\operatorname{Fr} D$

$$
\begin{aligned}
\liminf _{x \rightarrow y} H(x) & \geq \liminf _{x \rightarrow y}\left|I_{f, D}(x)\right| \\
& \geq \liminf _{x \rightarrow y}\left|I_{f, D}(x)\right|=|f(y)| \geq f^{+}(y) .
\end{aligned}
$$

Hence $H \in U_{f^{+}, D}$ and since $f^{+}$is continuous, it follows that $f^{+}$is resolutive (see case $f \geq 0)$. As (8.2) also holds when $f^{+}(y)$ is replaced by $f^{-}(y)$, it follows that $f^{-}$is also resolutive. Thus $f$ is resolutive i.e. a PWB solution exists.

Note that the "if" part of the theorem only uses the $L$-regularity of $D$.

We now show that $I_{f, D}$ and $H_{f, D}$ only differ by a singular harmonic function.

THEOREM 8.3. Let $D$ be an L-regular domain and $f$ a continuous function on $\operatorname{Fr} D$. Suppose that the PWB and an inner Dirichlet solution for $f$ exist. Then for any inner solution $I_{f, D}$ such that $I_{f, D} \leq I_{f^{+}, D}$ for some $I_{f^{+}, D}$ the function $I_{f, D}-H_{f, D}$ is singular in $D$.

Proof. Suppose first that $f \geq 0$. Then $I_{f, D}$ is in $U_{f, D}$ so that $I_{f, D}-H_{f, D}=s$, say, is nonnegative in $D$. Moreover, as $D$ is $L$-regular $s$ vanishes at all points of $\operatorname{Fr} D$ where $f$ is finite. Since $f$ is resolutive the complement of this set in $\operatorname{Fr} D$ is of zero harmonic measure. Thus, if $g$ is a nonnegative bounded harmonic minorant of $s$ in $D$ then $g=0$ in $D$. Hence $s$ is singular.

Again, if $f$ is of any sign we work with $f^{+}$and $f^{-}$. Since $f$ is resolutive, then PWB solutions for $f^{+}$and $f^{-}$exist. Thus, as $f^{+}$and $f^{-}$are continuous and $D$ is $L$-regular, it follows that $H_{f^{+}, D}$ and $H_{f^{-}, D}$ are inner Dirichlet solutions for $f^{+}$and $f^{-}$, respectively. Let $h_{1}$ be an inner solution for $f^{+}$such that $h_{1} \geq I_{f, D}$. We will now prove that the function 


$$
h_{1}-I_{f, D}-H_{f^{-}, D}=s_{1} \text {, say. }
$$

is singular in $D$. First, note that $\lim \left(h_{1}-I_{f, D}\right)=f^{-}$except at points where $f=$ $+\infty$. As $h_{1}-I_{f, D}$ is bounded below, it follows that $h_{1}-I_{f, D} \geq H_{f-, D}$. Thus $s_{1} \geq 0$ in $D$. Hence, as seen above, to show that $s_{1}$ is singular, it is enough to prove that $s_{1}$ vanishes on $\operatorname{Fr} D \backslash E$, where $E$ is of zero harmonic measure. But, if $E=\{y \in \operatorname{Fr} D:|f(y)|=\infty\}$, then it is easy to check that $s_{1}$ vanishes on $\operatorname{Fr} D \backslash E$. As $E$ is of zero harmonic measure the result follows. Also, by the first part of the proof, there exists a singular harmonic function $s_{2} \geq 0$ in $D$ such that

$$
h_{1}=H_{f^{+}, D}+s_{2}
$$

in $D$. Thus, using (8.4)

$$
\begin{aligned}
I_{f, D} & =\left(H_{f^{+}, D}+s_{2}\right)-H_{f^{-}, D}-s_{1} \\
& =H_{f, D}+\left(s_{2}-s_{1}\right)
\end{aligned}
$$

whence

$$
s=I_{f, D}-H_{f, D}=s_{2}-s_{1} .
$$

But

$$
|s| \leq\left|s_{1}\right|+\left|s_{2}\right|=s_{1}+s_{2} \text {. }
$$

Hence the least harmonic majorant $s^{*}$ of $|s|$ in $D$ exists. Further,

$$
s^{*} \leq s_{1}+s_{2}
$$

and $s_{1}$ and $s_{2}$ are singular. Hence $s_{1}+s_{2}$ is also singular whence $s^{*}$ is singular. This completes the proof of Theorem 8.4.

At this stage we must point out that we do not know whether any inner solution $I_{f, D}$ satisfies the condition of Theorem 8.3. What is certain and easy to establish is that the inequality $I_{f, D} \leq I_{f^{+}, D}$ does not hold for arbitrary $I_{f, D}$ and $I_{f^{+}, D}$.

\section{REFERENCES}

[1] K. Adachi, On the multiplicative Cousin problems for $N^{P}(D)$, Pacific J. Math. (2), 20 (1979), 297-303.

[2] D. H. Armitage, A strong type of regularity for the PWB solution of the Dirichlet problem, Proc. Amer. Math. Soc., 61 (1976), 285-289.

[ 3 ] F. T. Brawn, Positive harmonic majorization of subharmonic functions in strips, Proc. London Math. Soc. (3), 27 (1973), 261-289.

[4] M. Brelot, Sur la mesure harmonique et le problème de Dirichlet, Bull. Sci. Math. (2), 69 (1945) 153-156.

[5] M. Brelot, Etude générale des fonctions harmoniques ou surharmoniques positives 
au voisinage d'un point-frontière irrégulier, Ann. de l'université de (irenoble, Tome XXII, (1946) 205-219.

[6] M. Brelot, Remarques sur la variation des fonctions sousharmoniques et les masses associées. Application, Ann. Inst. Fourier, 2 (1950) 101-112.

$17 \mid$ B. E. J. Dahlberg, Estimates of harmonic measure, Arch. Rational Mech. Anal. (3), 65 (1.977) 275-288.

[8| J. L. Doob, "Classical Potential Theory and its Probabilistic Counterpart", Springer-Verlag, N-York, (1.984).

191 S. J. Gardiner, "Generalised means of subharmonic functions", Doctorate thesis, The Queen's University of Belfast, (1982).

[10] P. M. Gauthier and M. Goldstein, From local to global properties of subharmonic functions on Green spaces, J. London Math. Soc. (2), 16 (1977) 458-466.

111] D. S. Jeriaon and C. E. Kenig. Boundary behavior of harmonic functions in NonTangentially Accessible domains, Advances in Math., 46 (1982) 8()-147.

(12) U. Kuran, A criterion of harmonic majorization in half-spaces, Bull. London Math. Soc., 3 (1.971) $21-22$.

113] L. Naim, Sur le role de la frontière de R. S. Martin dans la théorie du potentiel. Ann. Inst. Fourier., (Grenoble), 7 (1957) 183-281.

114] Sawai Nualtarance, "On least harmonic majorants in half-spaces". Proc. London Math. Soc. (3), 27 (1973) $243-260$.

[15] E. D. Solomencev, Classes of functions subharmonic on a half-space, Vestnick Moskov. Univ. Ser. I. Mat. Meh., 5 (1959) 73-91.

[16] S. Zarantonello, The multiplicative Cousin Problem and a zero set for the Nevanlinna class on the polydisc, Trans. Amer. Math. Soc., 200 (1974) 291-313.

Departement de Mathematiques

Université de Tizi-()uzou

Algeria

current address:

2.3 Smollelt Street

Bootle

Liverpool L20 HPT

England 